\title{
Unusual MR Feature Presenting Discrete Involvement of Pyramidal Tract in Progressive Multifocal Leukoencephalopathy: A Case Report
}

\author{
Maki Umino ${ }^{1 *}$, Masayuki Maeda ${ }^{1}$, Nobuyoshi Matsushima ${ }^{1}$, Ai Itoh $^{2}$, \\ Akira Taniguchi $^{2}$, Hidekazu Tomimoto ${ }^{2}$ \\ ${ }^{1}$ Department of Radiology, Mie University School of Medicine, Tsu, Japan \\ ${ }^{2}$ Department of Neurology, Mie University School of Medicine, Tsu, Japan \\ Email: "m-tochio@clin.medic.mie-u.ac.jp
}

Received July 18, 2012; revised August 20, 2012; accepted August 31, 2012

\begin{abstract}
Progressive multifocal leukoencephalopathy (PML) is a demyelinating disease caused by reactivation of JC virus in immunocompromised patients. To date, PML with discrete involvement of the pyramidal tract has been described in only two patients. This report describes an additional case with PML showing discrete involvement of the pyramidal tract on T2-weighted images and FLAIR images.
\end{abstract}

Keywords: Progressive Multifocal Leukoencephalopathy; Pyramidal Tract; MRI

\section{Introduction}

Progressive multifocal leukoencephalopathy (PML), a disease of the central nervous system (CNS), is characterized by destructive infection of oligodendrocytes by JC virus [1]. Interference of the immune system, such as that by human immunodeficiency virus infection, malignancy or immunosuppressive drugs, reactivates the virus and might engender the disease.

The characteristic MRI appearance of PML is single or multifocal lesions that are round to oval at first and which become confluent and large with progression of the disease. The involvement of arcuate fibres creates a sharp border with the cortex. The lesions are hypointense on T1-weighted images and are hyperintense on T2weighted and FLAIR images. Lesions do not enhance and have no mass effect [2]. Although characteristic MR appearances have been reported in PML, dilemmas of differentiation from other diseases can occur based on imaging alone. This report describes a case presenting unusual MR features of discrete involvement of the pyramidal tract in light of the literature.

\section{Case Report}

A 61-year-old woman had suffered from liver cirrhosis and hepatic carcinoma for two years. She had also been treated for systemic lupus erythematosus (SLE) with steroids for one year. She developed progressive right hemi-

${ }^{*}$ Corresponding author. paresis followed by dementia with a history of two months. MR imaging was performed including T1-weighted imaging, FLAIR imaging, T2-weighted imaging, diffusionweighted imaging (DWI), contrast-enhanced T1-weighted imaging, and MR spectroscopy. The T2-weighted images showed high signal lesions in the left temporal lobe, fundamentally confined to white matter (Figure 1(A)). Almost no mass effect was found, considering the size of lesions. The lesions discretely involved the left pyramidal tract on the coronal FLAIR image (Figure 1(B)). T1weighted images showed slightly low signal and iso-signal intensities of lesions (Figure 1(C)). No enhancement was visible in any lesions on contrast-enhanced T1-weighted images. DWI showed partially high signal areas in lesions, particularly in the left pyramidal tract (Figure 1(D)). MR spectroscopy revealed an increased choline peak and decreased N-acetyl aspartate (NAA) (Figure 1(E)). PML and brain tumour such as lymphomatosis cerebri and gliomatosis cerebri were suspected as differential diagnoses based on MR imaging findings as well as clinical information.

The patient underwent brain biopsy followed by cerebrospinal fluid (CSF) polymerase chain reaction (PCR) analysis of JC virus. Stereotactic brain biopsy of the lesion from left temporal lobe showed no tumour cells but features of demyelination of the white matter with reactive astrocytic cell proliferation. Occasional oligodendrocytic and astrocytic cell nuclei had basophilic large inclusions (Figure 1(F)). CSF PCR analysis revealed JC 

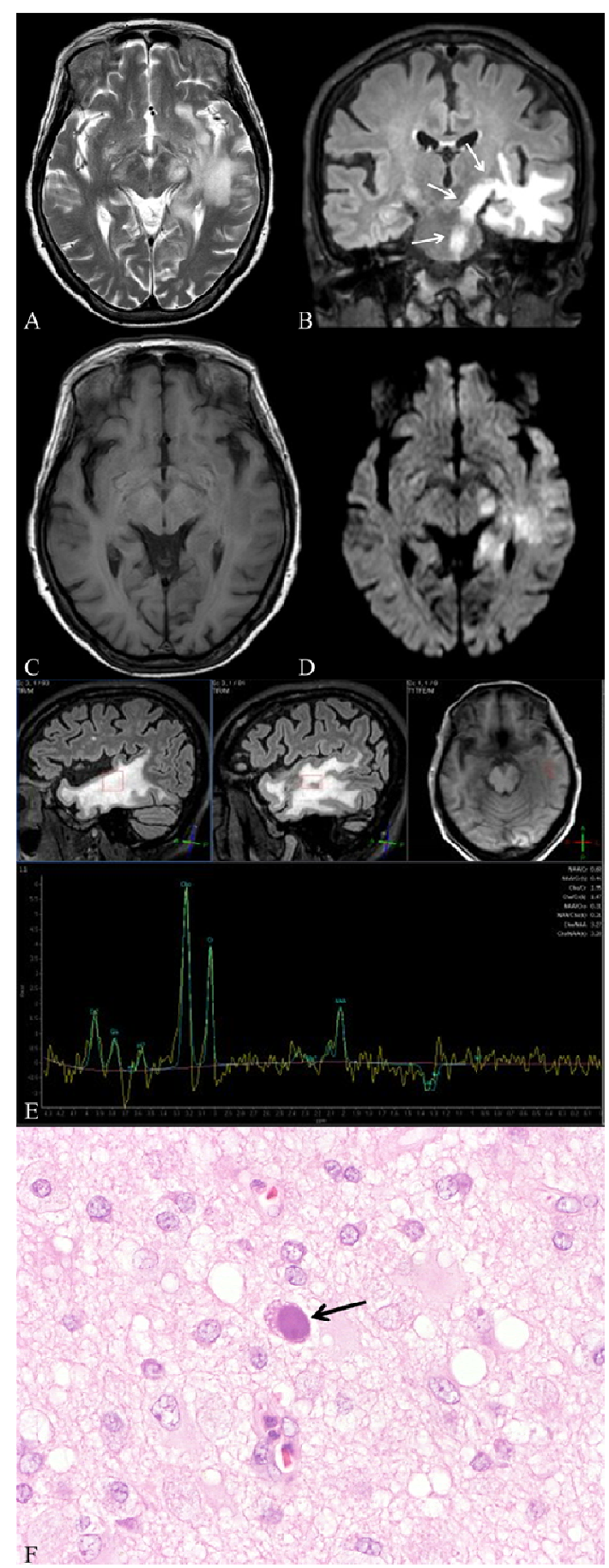

Figure 1. (A) Axial T2-weighted image shows high signal lesions in the left temporal lobe that are fundamentally confined to white matter; (B) Coronal FLAIR image shows a high signal lesion discretely involving the left pyramidal tract (arrows); (C) Axial T1-weighted image shows slightly low signal and iso-signal intensities of lesions; (D) DWI shows partially high signal areas in lesions, particularly in the left pyramidal tract; (E) MR spectroscopy shows an increased choline peak and decreased $\mathrm{N}$-acetyl aspartate; (F) JC virus-infected oligodendrocytes exhibit nuclear swelling and chromatin dissolution (arrow) (haematoxylin and eosin stain, $\times 400)$. virus infection leading to the diagnosis of PML. The patient was treated with mirtazapine. However, the patient's condition deteriorated thereafter. Serial MR imaging showed lesions extensively involving the cerebellum. Therefore, the patient was treated with mefloquine instead of mirtazapine, but she died after six months.

\section{Discussion}

PML is a fatal virally induced demyelinating disease that was once rare, but which has become more prevalent today. PML develops in patients with impaired immunity. The number of reported cases of this disease has increased substantially because of the pandemic of acquired immunodeficiency syndrome (AIDS). In non-AIDS patients, advancements in therapy of underlying diseases and the increasing use of immunomodulatory drugs have markedly changed the clinical background, particularly in patients with hematologic malignancy. The disease can also develop in patients with rheumatic diseases related or unrelated to immunosuppressive drug treatment; indeed, SLE is a common underlying disease [3]. Consequently, PML is now common and can develop in association with numerous different diseases, treatments, and conditions that engender immunodeficiency.

The characteristic MR appearance of PML is single or multifocal white matter lesions that become confluent and large with progressive disease. The involvement of $U$ fibres creates a sharp border with the cortex [2]. Usually, no mass effect or enhancement exists. High signal on DWI and low apparent diffusion coefficient (ADC) represent the regions of active infection and cell swelling [4]. MR spectroscopy shows elevated choline and reduced NAA [5]. In addition, our case was unique in that it showed discrete involvement of the pyramidal tract. To date, PML with a discrete involvement of the pyramidal tract had been described only in two cases [6,7]. All three cases including ours presented with rapidly progressive hemiparesis as the first clinical sign, presumably because of discrete involvement of pyramidal tract of PML. In our case, brain MR images showed diffuse white matter changes mimicking brain tumours such as gliomatosis cerebri and lymphomatosis cerebri. Those brain tumours and oligodendrogliomas are also known to show continuous involvement of the pyramidal tract on MR imaging or autopsy brain [8-10].

The MR imaging findings of gliomatosis cerebri are similar to those of PML, including widespread lesions with several lobes, no enhancement, and increased choline/NAA ratio [11-13]. Reportedly, gliomatosis cerebri shows slight effacement of the ventricles without displacement of the midline in $57.1 \%$ of cases and displacement of the ventricles with slight midline displacement in $42.9 \%$ of cases [11]. Consequently, it is noteworthy that the finding of slight effacement or the mass 
effect of ventricles is important for differential diagnosis between gliomatosis cerebri and PML.

Lymphomatosis cerebri is a rare variant of primary central nervous system lymphoma. Clinically, the disease typically presents with rapidly progressive dementia and unsteadiness of gait. Its presentation on cerebral MR imaging, which is characterized by diffuse leukoencephalopathy without contrast enhancement and mass effect, often causes diagnostic dilemmas with suspected diagnoses ranging from Binswanger disease to leukoencephalopathy or encephalomyelitis [8,14]. Findings of DWI and MR spectroscopy have not been reported yet for lymphomatosis cerebri.

Oligodendrogliomas are primary glial neoplasms derived from oligodendrocytes. Seizure has been the most common presenting symptom. To date, Oligodendroglioma with a discrete involvement of the pyramidal tract had been described only in one case [9]. Both the maximum ADC values and minimum ADC values were significantly different from normal brain. The $\mathrm{Cho} / \mathrm{Cr}$ is significantly higher than normal brain, and the NAA/Cr significantly lower than normal brain [15].

To conclude, we presented unusual MR features of discrete involvement of pyramidal tract in a patient with PML. This knowledge might help to produce correct diagnoses of this disease.

\section{REFERENCES}

[1] J. R. Berger and E. O. Major, "Progressive Multifocal Leukoencephalopathy," Seminars in Neurology, Vol. 19, No. 2, 1999, pp. 193-200. doi:10.1055/s-2008-1040837

[2] R. Shah, A. K. Bag, P. R. Chapman and J. K. Curé, “Imaging Manifestations of Progressive Multifocal Leukoencephalopathy,” Clinical Radiology, Vol. 65, No. 6, 2010, pp. 431-439. doi:10.1016/j.crad.2010.03.001

[3] E. J. Boren, G. S. Cheema, S. M. Naguwa, A. A. Ansari and M. E. Gershwin, "The Emergence of Progressive Multifocal Leukoencephalopathy (PML) in Rheumatic Diseases,” Journal of Autoimmunity, Vol. 30, No. 1-2, 2008, pp. 90-98. doi:10.1016/j.jaut.2007.11.013

[4] M. Bergui, G. B. Bradac, K. K. Oguz, A. Boghi, C. Geda, G. Gatti, et al., "Progressive Multifocal Leukoencephalopathy: Diffusion-Weighted Imaging and Pathological Correlations,” Neuroradiology, Vol. 46, No. 1, 2004, pp. 22-25. doi:10.1007/s00234-003-1115-9

[5] A. Iranzo, A. Moreno, J Pujol, J. Martí-Fàbregas, P. Domingo, J. Molet, et al., "Proton Magnetic Resonance Spectroscopy Pattern of Progressive Multifocal Leukoence- phalopathy in AIDS," Journal of Neurology, Neurosurgery \& Psychiatry, Vol. 66, No. 4, 1999, pp. 520-523. doi:10.1136/jnnp.66.4.520

[6] A. Semmler, H. Urbach, T. Klockgether and M. Linnebank, "Progressive Multifocal Leukoencephalopathy with Selective Involvement of the Pyramidal Tracts," Neurology, Vol. 68, No. 11, 2007, p. 871. doi:10.1212/01.wnl.0000257132.24299.76

[7] N. Sobha, S. Sinha, A. B. Taly, G. R. Arunodaya, S. Ravishankar, B. Anandh, et al., "Progressive Multifocal Leucoencephalopathy with Discrete Involvement of Pyramidal Tract," Journal of Neurology, Neurosurgery \& Psychiatry, Vol. 76, No. 1, 2005, p. 24.

doi:10.1136/jnnp.2004.046003

[8] K. E. Rollins, B. K. Kleinschmidt-DeMasters, J. R. Corboy, D. M. Damek and C. M. Filley, "Lymphomatosis Cerebri as a Cause of White Matter Dementia," Human Pathology, Vol. 36, No. 3, 2005, pp. 282-290. doi:10.1016/j.humpath.2005.01.014

[9] J. Pallud, B. Devaux, C. Daumas-Duport, C. Oppenheim and F. X. Roux, "Glioma Dissemination along the Corticospinal Tract,” Journal of Neuro-Oncology, Vol. 73, No. 3, 2005, pp. 239-240. doi:10.1007/s11060-005-0378-X

[10] E. Duron, A. Lazareth, J. Y. Gaubert, C. Raso, O. Hanon and A. S. Rigaud, "Gliomatosis Cerebri Presenting as Rapidly Progressive Dementia and Parkinsonism in an Elderly Woman: A Case Report," Journal of Medical Case Reports, Vol. 20, No. 2, 2008, p. 53. doi:10.1186/1752-1947-2-53

[11] A. Yu, K. Li and H. Li, "Value of Diagnosis and Differential Diagnosis of MRI and MR Spectroscopy in Gliomatosis Cerebri,” European Journal of Radiology, Vol. 59, No. 2, 2006, pp. 216-221. doi:10.1016/j.ejrad.2006.03.001

[12] K. Sakushima, M. Yashima-Yamada, K. Kubota, Y. Sawamura, I. Yabe and H. Sasaki, "Gliomatosis Cerebri with Multifocal Progressive Lesions on MRI," Rinsho Shinkeigaku, Vol. 51, No. 2, 2011, pp. 145-148. doi:10.5692/clinicalneurol.51.145

[13] R. del Carpio-O’Donovan, I. Korah, A. Salazar and D. Melançon, "Gliomatosis Cerebri,” Radiology, Vol. 198, No. 3, 1996, pp. 831-835.

[14] S. Terae and A. Ogata, "Nonenhancing Primary Central Nervous System Lymphoma,” Neuroradiology, Vol. 38, No. 1, 1996, pp. 34-37. doi:10.1007/BF00593213

[15] M. D. Jenkinson, T. S. Smith, A. R. Brodbelt, K. A. Joyce, P. C. Warnke and C. Walker, “Apparent Diffusion Coefficients in Oligodendroglial Tumors Characterized by Genotype,” Journal of Magnetic Resonance Imaging, Vol. 26, No. 6, 2007, pp. 1405-1412. doi:10.1002/jmri.21062 